Published in final edited form as:

Nat Clim Chang. 2018 June ; 8(6): 510-514. doi:10.1038/s41558-018-0161-6.

\title{
Antibiotic Resistance Increases with Local Temperature
}

\author{
Derek R. MacFadden ${ }^{\star}, 1,2,3$, Sarah F. McGough ${ }^{\# 2,4}$, David Fisman ${ }^{1}$, Mauricio \\ Santillana ${ }^{3,4,5, \psi}$, and John S. Brownstein ${ }^{3,4,5, \psi}$ \\ ${ }^{1}$ Division of Infectious Diseases, Department of Medicine, University of Toronto, Canada. \\ ${ }^{2}$ Harvard Chan School of Public Health, Harvard University, Boston, United States. \\ ${ }^{3}$ Computational Epidemiology Group, Boston Children's Hospital, Boston, United States. \\ ${ }^{4}$ Computational Health Informatics Program, Boston Children's Hospital, Boston, United States. \\ ${ }^{5}$ Department of Pediatrics, Harvard Medical School, Harvard University, Boston, United States. \\ \# These authors contributed equally to this work.
}

Bacteria that cause infections in humans can develop or acquire resistance to antibiotics commonly used against them ${ }^{1,2}$. Antimicrobial resistance causes significant morbidity worldwide, and some estimates indicate attributable mortality could reach up to 10 million by $2050^{2-4}$. Antibiotic resistance in bacteria is believed to develop largely under the selective pressure of antibiotic use; however, other factors may contribute to population level increases in antibiotic resistance ${ }^{1,2}$. We explored the role of climate (temperature) and additional factors on the distribution of antibiotic resistance across the United States, and here we show that increasing local temperature as well as population density were associated with increasing antibiotic resistance (percent resistant) in common pathogens. We found that an increase in temperature of $10^{\circ} \mathrm{C}$ across regions was associated with increases in antibiotic resistance of $4.2 \%, 2.2 \%$, and $2.7 \%$ for the common pathogens Escherichia coli, Klebsiella pneumoniae, and Staphylococcus aureus. The associations between temperature and antibiotic resistance in this ecologic study were consistent across most classes of antibiotics and pathogens and may be strengthening over time. These findings suggest that current forecasts of the burden of antibiotic resistance could be significant underestimates in the face of a growing population and climate change 4 .

Since the discovery of Penicillin by Alexander Fleming in 1928, antibiotics have had immeasurable impact on the reduction of bacterial infections and associated morbidity and

\footnotetext{
*Correspondence to: Derek R. MacFadden, MD FRCPC, 200 Elizabeth Street 13EN-213, University Health Network, Toronto ON Canada, M5G 2C4, Tel: 416 340-4410, Fax: 416 340-3357, derek.macfadden@ mail.harvard.edu. Mauricio Santillana, PhD, One Autumn Street, AU 451, Boston Children's Hospital, Boston, MA 02215 Tel: 617 599-5460, Fax: 617-919-1795, msantill@g.harvard.edu,. ${ }^{\psi}$ Co-Principal Investigators.

Author Contributions

DM, SM, and MS contributed to the data-analysis. All authors (DM, SM, DF, MS, JB) contributed to development of the manuscript, discussion, and preparation of final versions. All authors approved the final version of the manuscript.

Author Information

All authors have no relevant competing risks to declare. Correspondence and requests for materials should be addressed to DM (derek.macfadden@mail.harvard.edu).

All authors have read/approved the manuscript. This manuscript has not been published elsewhere. No writing assistance was provided in preparation. Data used in the study are from openly available sources and are described/referenced in the supplemental materials
} 
mortality ${ }^{1,2}$. However, their global use (and overuse) has supported the spread of bacterial resistance to these agents ${ }^{1,2}$, with the possibility of entering a 'post-antibiotic era' in which antibiotics are no longer a treatment option ${ }^{2-4}$. Despite the potential dire implications for human health posed by antibiotic resistance over the next century, we have a limited understanding of the factors that drive the transmission of antibiotic resistance amongst populations, including the potential impact of climate change ${ }^{1,5}$.

Antibiotic resistance can spread by the horizontal transmission of specific genomic resistance mechanisms and the selection/propagation of resistant strains ${ }^{1,6}$, and this is known to occur (often asymptomatically) between humans, animals, and the environment ${ }^{1}$ (we will collectively refer to this movement as 'transmission'). While antibiotic use has been assumed to be the main force behind the rise of antibiotic resistance in populations, other factors may act to facilitate transmission of antibiotic resistance and drive regional spread ${ }^{1,7}$. Temperature has long been known to affect bacterial growth in-vitro as well as modulate transfer of genomic material, including genes encoding (or conferring) antibiotic resistance ${ }^{8,9}$, but empirical evidence of the impact of climate on population level resistance is lacking. Here, we investigated whether local climate, in the form of local temperature, could explain differences in antibiotic resistance across geographic regions in the United States at high spatial resolution. In addition, we sought to evaluate whether local characteristics such as population density, antibiotic prescription rates, specific patient populations, and laboratory standards could also account for observed differences in antibiotic resistance patterns.

We developed a large database of antibiotic resistance patterns across different geographic regions in the United States using antibiotic resistance indices (index of resistance by antibiotic and bacterial pathogen) generated from information sourced from hospitals, laboratories, and surveillance units ${ }^{10}$. Resistance indices were linked with facility characteristics, such as inpatient/outpatient type, as well as regional prescribing rates ${ }^{11}$ and population density ${ }^{12}$. We studied three common community and hospital-acquired bacterial pathogens, including two Gram-negative species (Escherichia coli and Klebsiella pneumoniae) and one Gram-positive species Staphylococcus aureus ${ }^{13,14}$. The final dataset represented over 1.6 million clinically relevant bacterial pathogens, from 602 unique indices, spanning 223 facilities, across 41 states, for the years 2013-2015.

We explored the relationships between regional patterns of antibiotic resistance (here defined as percent of pathogens not susceptible to a particular antibiotic) and both latitude and local historical climate variables (mean and minimum temperature, averaged over 19802010) (Supplementary Fig. 1) ${ }^{15}$. Historical climate variables were chosen in order to match best with the periods of time over which antibiotic resistance patterns have developed. Average minimum temperature showed higher correlations than average temperature and latitude across the three pathogens and antibiotics (Supplementary Fig. 1), with correlations up to 0.7 . Minimum temperature is a commonly used climate variable when describing the survival of species within environments ${ }^{16}$ and may be of particular importance in identifying: (1) regions that can support persistence of environmental and/or colonizing bacteria; and (2) regions with enhanced growth and resistance transmission potential. Given these factors, and the relative strength of its relationship with antibiotic resistance, we 
focused our analysis on the association between minimum temperature and antibiotic resistance.

Using both unadjusted and multivariable adjusted weighted linear models, we evaluated multiple aspects of the association between minimum temperature and antibiotic resistance. We found that increasing minimum temperature was associated with increasing antibiotic resistance, and this was consistent across most classes of antibiotics and pathogens (Figs. 12, Supplementary Figs. 2-4). In unadjusted analysis (Table 1), an increase of $10{ }^{\circ} \mathrm{C}$ across regions was associated with an increase in antibiotic resistance of $5.1 \%(\mathrm{p}<0.0001), 3.4 \%$ ( $\mathrm{p}<0.0001$ ), and $3.1 \%$ ( $\mathrm{p}=0.002$ ) for E.coli, K. pneumoniae, and S. aureus, respectively. Notably, changes in minimum temperature lead to larger increases in antibiotic resistance in subsequent years of observations, when analysing all antibiotics and pathogens, both for oral and intravenous (IV) formulations (Fig. 2). The measure of association between minimum temperature and antibiotic resistance may increase with earlier time of introduction in the United States (Supplementary Fig. 5). The geographic pattern of antibiotic resistance in the common pathogen E.coli is shown in Fig. 1, and in Supplementary Figs. 3-4 for $K$. pneumoniae and $S$. aureus respectively.

Multivariable analysis of antibiotic resistance versus minimum temperature was performed for each pathogen across all tested antibiotics (Table 1). After adjusting for acquisition source, prescription rate (available for a subset of tested antibiotics), population density, and laboratory standard, we found that a $10{ }^{\circ} \mathrm{C}$ increase in minimum temperature across regions was associated with an increase in antibiotic resistance of $4.2 \%$ ( $\mathrm{p}<0.0001$ ), $2.2 \%$ ( $<<0.0001$ ), and $2.7 \%$ ( $\mathrm{p}=0.21$ ) for E. coli, K. pneumoniae, and S. aureus, respectively. When we removed penicillin (an uncommon and sporadically tested antibiotic) susceptibility results for $S$. aureus, we found that minimum temperature was associated with an increase in antibiotic resistance of $5.1 \%(\mathrm{p}<0.0001)$ (Table 1$)$. We also looked at $S$. aureus resistance to specific antibiotics including cloxacillin, an older antibiotic which is used to differentiate methicillin-susceptible from methicillin-resistant $S$. aureus (MRSA). In multivariable adjusted analysis, we found increases in antibiotic resistance to cloxacillin, fluoroquinolones, and macrolides, of 5.8\% ( $\mathrm{p}<0.0001), 3.7 \%(\mathrm{p}=0.096)$, and $6.0 \%(\mathrm{p}<0.001)$ respectively for a $10{ }^{\circ} \mathrm{C}$ increase in minimum temperature across regions (Supplementary Table 1). These multivariable results generally support the univariate associations seen.

Geographic variability in antibiotic prescribing has been documented in outpatient facilities between broad regions within the United States ${ }^{17,18}$, where an increased incidence of antibiotic prescriptions has been observed across a number of southern states ${ }^{17}$. Given these patterns, we examined the effects of spatial distributions of antibiotic prescribing on resistance for four common antibiotic classes penicillins, cephalosporins, fluoroquinolones, and macrolides. We found that increased prescribing was associated with increasing antibiotic resistance for the antibiotics evaluated across all three pathogens (Supplementary Fig. 6, Table 1). Moreover, the relationship between minimum temperature and antibiotic use did not change by amount (tertile) of antibiotic prescribing (Supplementary Fig. 7).

In multivariable adjusted analyses (Table 1) we found that an increase in population density by 10,000 persons per square mile (e.g. from Bismarck, North Dakota to Boston, 
Massachusetts) was associated with a $6 \%$ increase in antibiotic resistance for $K$. pneumoniae ( $\mathrm{p}<0.0001)$ and $3 \%$ for $E$. coli $(\mathrm{p}=0.086)$. This may reflect increased transmission rates within dense populations ${ }^{19}$. Sources containing outpatient antibiotic resistance data were associated with lower resistance for all pathogens, and this association was significant in adjusted models for $S$. aureus $(-4.24 \%, \mathrm{p}=0.0003)$, but not for $E$. coli $(-2.98 \%, \mathrm{p}=0.06)$ or

K. pneumoniae $(-2.88 \%, \mathrm{p}=0.17)$ (Table 1$)$. Reported laboratory standard only demonstrated a significant association with antibiotic resistance in the adjusted analyses of $S$. aureus isolates. We found no change in the relationship between minimum temperature and antibiotic resistance across levels of the other evaluated covariates (Supplementary Fig. 7). Furthermore, we found no strong correlations between minimum temperature and other covariates (Supplementary Table 2).

While the mechanisms underlying the observed associations between resistance and temperature require further elucidation, several hypotheses are proposed. Firstly, temperature may facilitate horizontal gene transfer, occurring through exchange of resistance genes (e.g. plasmid-borne extended spectrum-beta lactamases - ESBLs) or uptake of free genetic material ${ }^{8,9,20}$. European countries in the southern latitudes have a higher incidence of infections due to ESBL producing enterobacteriaceae ${ }^{21}$, which has generally been attributed to antibiotic use and selective pressure, but which may be facilitated by climate factors including temperature. Many recent emergences of highly mobile genetic elements of resistance have originated from central latitudes ${ }^{9,22,23}$. Increased gene transfer (occurring either at sites of colonization on a host ${ }^{24}$, or in the environment ${ }^{9}$ ), might then be expected to facilitate population transmission. Secondly, temperature is one of the most potent modifiers of bacterial growth rates ${ }^{24,25}$, and may drive increased carriage ${ }^{26}$ and transmission of resistant strains of between humans and animals. For both Gram-positive and Gram-negative human pathogens, seasonal patterns of infection have been identified and carriage may play a role 27,28 . In a similar fashion, temperature may support environmental growth of resistant strains leading to enhanced transmission from food/agriculture and environmental sources 29. Increased population level transmission may thus facilitate population level selection of resistant strains ${ }^{30}$. Interestingly, we also found that fluoroquinolones and beta-lactams generally demonstrated the strongest associations between temperature and resistance (Figs. 1, Supplementary Figs. 3-4). This would support mechanism specific impacts of temperature on resistance. Lastly, potential temperature effects may be rooted in more complex factors (e.g. behavioural, social) occurring across humans, animals, and agriculture/ environment, as embodied by the OneHealth perspective.

While a major limitation of our approach is that we compare climate and predictor data with antibiotic resistance at regional levels, and thus cannot infer causality, our approach is suited to identifying ecological associations which may be particularly relevant for antibiotic resistance (e.g. antibiotic prescriptions across population vs. individual) and is a commonly used design when studying antibiotic resistance over large populations and geographies ${ }^{21}$.

The World Health Organization has identified climate change as a major driver of emerging infectious diseases globally ${ }^{5}$, though vector-borne and enteric infections such as cholera have typically been identified as most likely to be impacted. Our findings suggest that the spread of antibiotic resistance may be modified and potentially accelerated by regional 
temperature and future climate change. Based upon our findings, a $10^{\circ} \mathrm{C}$ increase in temperature, a conceivable scenario for parts of the United States by the end of this century ${ }^{31}$, could yield additional increases in resistance on the order of $10 \%$ for certain antibiotics (Fig. 1, Supplementary Table 1). If the relationship between minimum temperature and antibiotic resistance is indeed present and increasing over time, this could support a more rapid progression towards a 'post-antibiotic era'. Further research is needed to confirm these relationships and elucidate the role of climate in antibiotic resistance spread. Our findings would suggest that in the presence of climate change and population growth, already dire predictions of the impact of antibiotic resistance on global health may be significant underestimates ${ }^{4}$.

\section{Methods}

\section{Patterns of Antibiotic Resistance}

To identify regional patterns of antibiotic resistance across the United States, we utilized a continuously-updated database of spatially localized annual indices of inpatient and outpatient antibiotic resistance for common clinically relevant bacterial pathogens from human clinical isolates for years $2013-2015^{10}$. Data entry into this database was performed by analysts blind to the research questions evaluated in this study. Each antibiotic resistance index corresponded to a particular health/surveillance network, hospital, or laboratory facility. Relevant variables captured in this database include antibiotic susceptibility, bacterial species, year, location, acquisition sources (inpatient only or containing outpatient isolates), and laboratory standard (clinical laboratory standards institute [CLSI] or other/not reported). Percent antibiotic resistance was defined as the percentage of bacterial isolates that were non-susceptible to a given antibiotic. Minimum inhibitory concentration (MIC) values were not available/evaluated in this dataset. Antibiotics evaluated for each pathogen are shown in Supplementary Table 3, including classification as intravenous (IV) formulation or available in a regularly used oral formulation. For specific drugs where testing of intravenous versions approximate susceptibility for oral equivalents, we substituted the oral drug name (which are more commonly used). These included amoxicillin and cephalexin in lieu of ampicillin and cefazolin. We restricted our analysis to the continental United States and focused on three important pathogens in community and nosocomial infections, Escherichia coli, Klebsiella pneumoniae, and Staphylococcus aureus 13,14. The distribution of pathogen specific data points are shown in Supplementary Fig. 8. After restricting to these three bacterial species, the final dataset represented over 1.6 million bacterial pathogens corresponding to up to 22.8 million susceptibility test results, from 602 unique indices, spanning 223 facilities, across 41 states throughout the continental United States. The number of indices contributing data for the pathogens E. coli, K. pneumoniae, and $S$. aureus were 595, 551 and 307 respectively, representing approximately 1.1 million, 0.2 million, and 0.3 million bacterial pathogens tested, respectively. Of this dataset, mean population density was 2,462 persons $/ \mathrm{mi}^{2}, 77.4 \%$ of indices contained outpatient data, and $70.8 \%$ of indices reported using CLSI standards (versus other/not reported). For analysis of the impact of prescription rates, we used a subset of the data based on availability of prescribing rates for relevant drug classes (fluoroquinolones, cephalosporins, penicillins, and macrolides), and this subset contained 446 indices, across 40 states, totaling 1.3 million 
bacterial pathogens, where mean prescribing rate was 139.8 prescriptions per 1,000 persons, mean population density was 2,583 persons $/ \mathrm{mi}^{2}, 78 \%$ of indices contained outpatient data, and $70 \%$ of indices reported using CLSI standards (versus other/not reported). A validation assessment of these datasets was performed, with a review of antibiotic resistance and descriptor data values randomly selected across indices from a 10\% sample of facilities included in the database, and yielded a concordance of $97 \%$ with original data sources.

\section{Predictors and Confounders}

We selected relevant predictors and confounders that help predict antibiotic resistance based on previous literature and plausible biologic mechanisms $1,13,18,25,28,32$. We linked each location by nearest zip code with climate data, including 30-year normals (1980-2010) of minimum temperature (30-year average of daily minimum temperature) and mean temperature (30-year average of mean daily temperature) from the United States National Climatic Data Center ${ }^{15}$. To account for spatial variability in antibiotic use, outpatient antibiotic prescriptions (annual prescriptions per 1000 persons) by class (fluoroquinolones, cephalosporins, penicillins, and macrolides) for the years 2013-2014 were linked to index location by antibiotic tested at the state-level, using data derived from Xponent database from QuintilesIMS (Danbury, Connecticut) via CDC antibiotic Resistance Patient Safety Atlas ${ }^{11}$. We assume that current distributions of antibiotic prescription rates are reflective of recent historical distributions as complete prescription data over a potentially relevant time course is not reasonably identifiable/attainable. Moreover, we assume that the prescribing measures and linear relationship we use adequately approximates the underlying relationships, and that we have captured the most relevant predictors/confounders. Finally, we linked the data with population density throughout the United States by zip code based on 2010 census data ${ }^{12}$.

\section{Data Analysis}

For all figures and analyses in this ecologic study, we used location/facility specific data points, prepared by weighted averages of antibiotic resistance indices by organism, drug, acquisition sources (inpatient or outpatient containing), and year, for the particular location. Where applicable, data markers were scaled proportional to the total number of isolates tested for a given organism at a particular location/facility and this was denoted in the legends. For ease of trend visualization of antibiotic resistance across multiple different antibiotics, we centered the data about the mean and normalized by the standard deviation (Fig. 2, Supplementary Figs. 2, 6-7). Trend bars represent weighted linear fitted models with standard confidence regions in grey. The effect of minimum temperature on antibiotic resistance was plotted within levels of the covariates (prescription rate - tertile, acquisition location, laboratory standard, and population density - tertile) in order to qualitatively assess for potential confounding and effect modification (Supplementary Fig. 7). Log scales were utilized for population density given the large spread of values (Supplementary Fig. 6). All plots were generated using R (Version 3.3.2, Vienna, Austria).

We generated heatmaps of normalized antibiotic resistance (Fig. 1, Supplementary Figs. 34) for each pathogen, based on average normalized resistance within zip codes containing antibiotic resistance data. We utilized reference 30-year minimum temperature normals for 
these same zip codes to generate the minimum temperature map (Fig. 1, Supplementary Figs. 3-4). Gaussian kernel smoothing was performed with least-squares cross-validated bandwidth selection. Map projections were obtained using the R packages "maptools" and "maps," and visualizations were performed using the package "spatstat.".

We used weighted linear regression of location specific antibiotic resistance with clustering by state and latitude quintile to account for correlated errors, and determined unadjusted and adjusted model parameters for relevant predictors (Table 1). Weighting was based on the total number of isolates tested for a given pathogen. All analyses were performed using $\mathrm{R}$ (Version 3.3.2, Vienna, Austria).

\section{Sensitivity Analyses}

In order to assess the potential impact of possible 'double counting' that could occur in regions where a large surveillance body may overlap with a hospital or laboratory, we evaluated the association of minimum temperature and antibiotic resistance using multivariable weighted regression, but restricted to the subset of health system, hospital, and laboratory data only. There were no major changes in overall interpretation of the data, and the results are shown in Supplementary Table 4.

To evaluate whether time trends in effect of minimum temperature on antibiotic resistance could be due to non-random sampling, we applied the same multivariable regression analysis as described in the main paper, but restricted to institutions that provided data for more than one year. There were no major changes in overall interpretation of the data, and the multivariable model results are shown in Supplementary Table 4.

To assess whether different isolate sources could impact the association between antibiotic resistance and temperature, we ran an additional multivariable linear weighted regression models, with the same parameters as the full model in Table 1 (minimum temperature, prescription rate, outpatient containing, laboratory standard, and population density) but also included binary predictors of isolate sources contained within the indices, namely: Urine; Blood; Respiratory; Other sterile (e.g. cerebrospinal fluid, pleural fluid, peritoneal fluid); and Other non-sterile (e.g. wound swabs). In order to accommodate these additional predictors, this model was run in the dataset prior to collapsing across the variables of interest. There were no major changes in overall interpretation of the data, and the results are shown in Supplementary Table 4.

To evaluate whether socioeconomic status could have an impact on the association between antibiotic resistance and temperature, we ran additional multivariable weighted linear regression models, with the same parameters as the full model, but with either the replacement of population density with median income or addition of median income (Supplemental Table 4). We found no significant changes to the overall model findings, although median income may be a relevant factor for E.coli resistance. Median income data was extracted from the US Census Bureau American Census Survey (ACS), 5-year estimate from 2015, and linked by zipcode. To evaluate the possible impact of imputation of cephalexin susceptibility on cloxacillin results, we ran additional multivariable weighted 
linear regression models for $S$. aureus with cephalexin susceptibilities removed. We found no major changes to the overall interpretation of the results (Supplementary Table 4).

\section{Limitations}

As previously noted, due to the nature of this descriptive ecologic study, and potential for ecologic bias, we cannot infer causality. We have selected confounders based on their potential relevance in antibiotic resistance transmission, however residual/unmeasured confounding is possible. Selecting important factors governing population level distribution of antibiotic resistance is challenging, as they are generally poorly understood. We also only included prescribing rates for antibiotic classes matching the antibiotic susceptibility and did not consider selection effects of other classes. Lastly, our dataset may be subject to selection bias, although, it is unlikely that minimum temperature would be associated with inclusion into the dataset.

\section{Data Availability}

Antibiotic resistance data is derived from open sources and is queryable at www.resistanceopen.com ${ }^{10}$. Climate data is available through the United States National Climatic Data Center ${ }^{15}$. Antibiotic prescription rates are available from Xponent database from QuintilesIMS (Danbury, Connecticut) via CDC antibiotic Resistance Patient Safety Atlas $^{11}$. Population density values are available through 2010 census data ${ }^{12}$.

\section{Supplementary Material}

Refer to Web version on PubMed Central for supplementary material.

\section{Acknowledgements}

DM is supported by a Canadian Institutes for Health Research Fellowship Grant and the Clinician Scientist Program at the Department of Medicine, University of Toronto. JB is supported by the National Library of Medicine NIH R01 LM011965. Thank you to the developers and data analysts at HealthMap for their support. Thank you to Moritz Kramer for his thoughtful and insightful review/feedback.

\section{References}

1. Holmes AH et al. Understanding the mechanisms and drivers of antimicrobial resistance. Lancet 387, 176-187 (2016). [PubMed: 26603922]

2. Nathan C \& Cars O Antibiotic Resistance - Problems, Progress, and Prospects. N. Engl. J. Med 371, 1761-1763 (2014). [PubMed: 25271470]

3. World Health Organization. Global Action Plan on Antimicrobial Resistance (2015).

4. O’Neill J Tackling Drug-Resistant Infections Globally: Final Report and Recommendations 2014. HM Government and Wellcome Trust: UK (2016).

5. The World Health Organization. Climate Change and Infectious Diseases Available at: http:// www.who.int/globalchange/environment/en/chapter6.pdf (Accessed: 27th March 2017)

6. Feero WG, Guttmacher AE \& Relman DA Microbial Genomics and Infectious Diseases. N. Engl. J. Med 365, 347-357 (2011). [PubMed: 21793746]

7. Wellington EMH et al. The role of the natural environment in the emergence of antibiotic resistance in Gram-negative bacteria. Lancet Infect. Dis 13, 155-165 (2013). [PubMed: 23347633]

8. Lorenz MG \& Wackernagel W Bacterial gene transfer by natural genetic transformation in the environment. Microbiol. Rev 58, 563-602 (1994). [PubMed: 7968924] 
9. Walsh TR, Weeks J, Livermore DM \& Toleman MA Dissemination of NDM-1 positive bacteria in the New Delhi environment and its implications for human health: an environmental point prevalence study. Lancet Infect. Dis 11, 355-362 (2011). [PubMed: 21478057]

10. MacFadden DR et al. A Platform for Monitoring Regional Antimicrobial Resistance, Using Online Data Sources: ResistanceOpen. J. Infect. Dis 214, S393-S398 (2016). [PubMed: 28830108]

11. Centers for Disease Control and Prevention. Antibiotic Resistance Patient Safety Atlas (2016). Available at: https://gis.cdc.gov/grasp/PSA/indexAU.html (Accessed: January 2017)

12. General Population and Housing Characteristics: 2010 Demographic Profile. US Census Bureau Available at: https://factfinder.census.gov/.

13. Peleg AY \& Hooper DC Hospital-Acquired Infections Due to Gram-Negative Bacteria. N. Engl. J. Med 362, 1804-1813 (2010). [PubMed: 20463340]

14. Boucher HW et al. Bad Bugs, No Drugs: No ESKAPE! An Update from the Infectious Diseases Society of America. Clin. Infect. Dis 48, 1-12 (2009). [PubMed: 19035777]

15. NOAA's 1981-2010 U.S. Climate Normals: An Overview. Bull. Am. Meteorol. Soc 93, 16871697 (2012).

16. Sutherst RW Global change and human vulnerability to vector-borne diseases. Clin. Microbiol. Rev 17, 136-173 (2004). [PubMed: 14726459]

17. Hicks LA, Taylor TH, Jr \& Hunkler, R. J. U.S. outpatient antibiotic prescribing, 2010. N. Engl. J. Med 368, 1461-1462 (2013). [PubMed: 23574140]

18. Hicks LA et al. US outpatient antibiotic prescribing variation according to geography, patient population, and provider specialty in 2011. Clin. Infect. Dis 60, 1308-1316 (2015). [PubMed: 25747410]

19. Hilty $\mathrm{M}$ et al. Transmission dynamics of extended-spectrum $\beta$-lactamase--producing Enterobacteriaceae in the tertiary care hospital and the household setting. Clin. Infect. Dis 55, 967-975 (2012). [PubMed: 22718774]

20. Warnes SL, Highmore CJ \& Keevil CW Horizontal transfer of antibiotic resistance genes on abiotic touch surfaces: implications for public health. MBio 3, (2012).

21. Goossens H, Ferech M, Vander Stichele R, Elseviers M \& ESAC Project Group. Outpatient antibiotic use in Europe and association with resistance: a cross-national database study. Lancet 365, 579-587 (2005). [PubMed: 15708101]

22. Poirel L, Potron A \& Nordmann P OXA-48-like carbapenemases: the phantom menace. J. Antimicrob. Chemother 67, 1597-1606 (2012). [PubMed: 22499996]

23. Liu Y-Y et al. Emergence of plasmid-mediated colistin resistance mechanism MCR-1 in animals and human beings in China: a microbiological and molecular biological study. Lancet Infect. Dis 16, 161-168 (2016). [PubMed: 26603172]

24. Shah HN \& Gharbia SE The Impact of the Environment on Human Infections. Microb. Ecol. Health Dis 11, 248-254 (1999).

25. Ratkowsky DA, Olley J, Mcmeekin TA \& Ball3, A. A. Relationship Between Temperature and Growth Rate of Bacterial Cultures. J. Bacteriol 149, 1-5 (1982). [PubMed: 7054139]

26. Dipl-Vw KK, Frank U, Conrad A \& Meyer E Seasonal and Ascending Trends in the Incidence of Carriage of Extended-Spectrum $\beta$-Lactamase-Producing Escherichia coli and Klebsiella Species in 2 German Hospitals. Infect. Control Hosp. Epidemiol 31, 1154-1159 (2010).

27. Perencevich EN et al. Summer Peaks in the Incidences of Gram-Negative Bacterial Infection Among Hospitalized Patients. Infect. Control Hosp. Epidemiol 29, 1124-1131 (2008).

28. Mermel LA, Machan JT, Parenteau S, Brown SM \& Jones K Seasonality of MRSA Infections. PLoS One 6, e17925 (2011). [PubMed: 21468350]

29. Gautam R et al. Modeling the effect of seasonal variation in ambient temperature on the transmission dynamics of a pathogen with a free-living stage: Example of Escherichia coli O157:H7 in a dairy herd. Prev. Vet. Med 102, 10-21 (2011). [PubMed: 21764472]

30. Levin BR Minimizing potential resistance: a population dynamics view. Clin. Infect. Dis 33 Suppl 3, S161-9 (2001). 
31. Intergovernmental Panel on Climate Change. Climate Change 2013: The Physical Science Basis: Working Group I Contribution to the Fifth Assessment Report of the Intergovernmental Panel on Climate Change (Cambridge University Press, 2014).

32. Bruinsma $\mathrm{N}$ et al. Influence of population density on antibiotic resistance. J. Antimicrob. Chemother 51, 385-390 (2003). [PubMed: 12562707] 

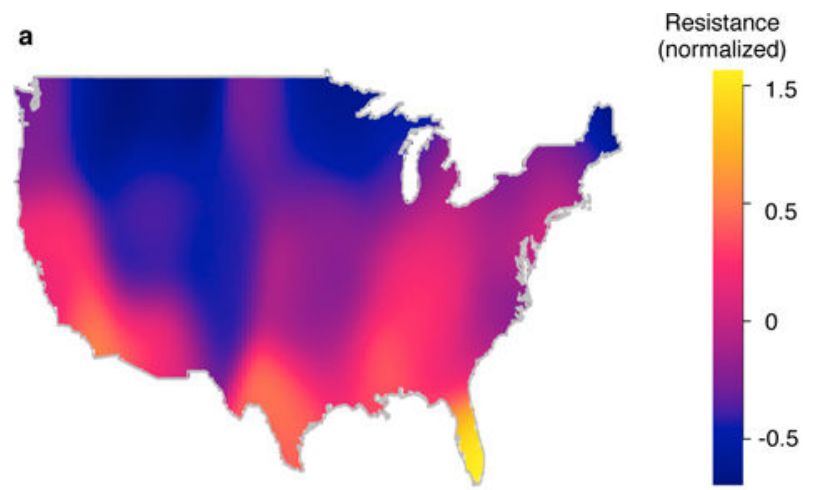

b

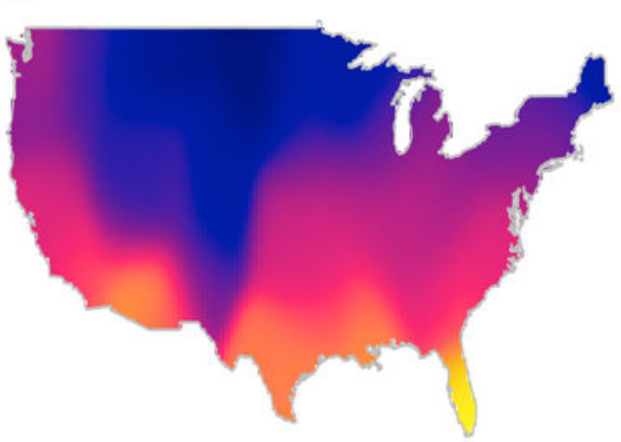

Minimum temp

$\left({ }^{\circ} \mathrm{C}\right)$

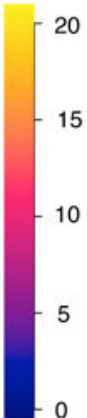

C
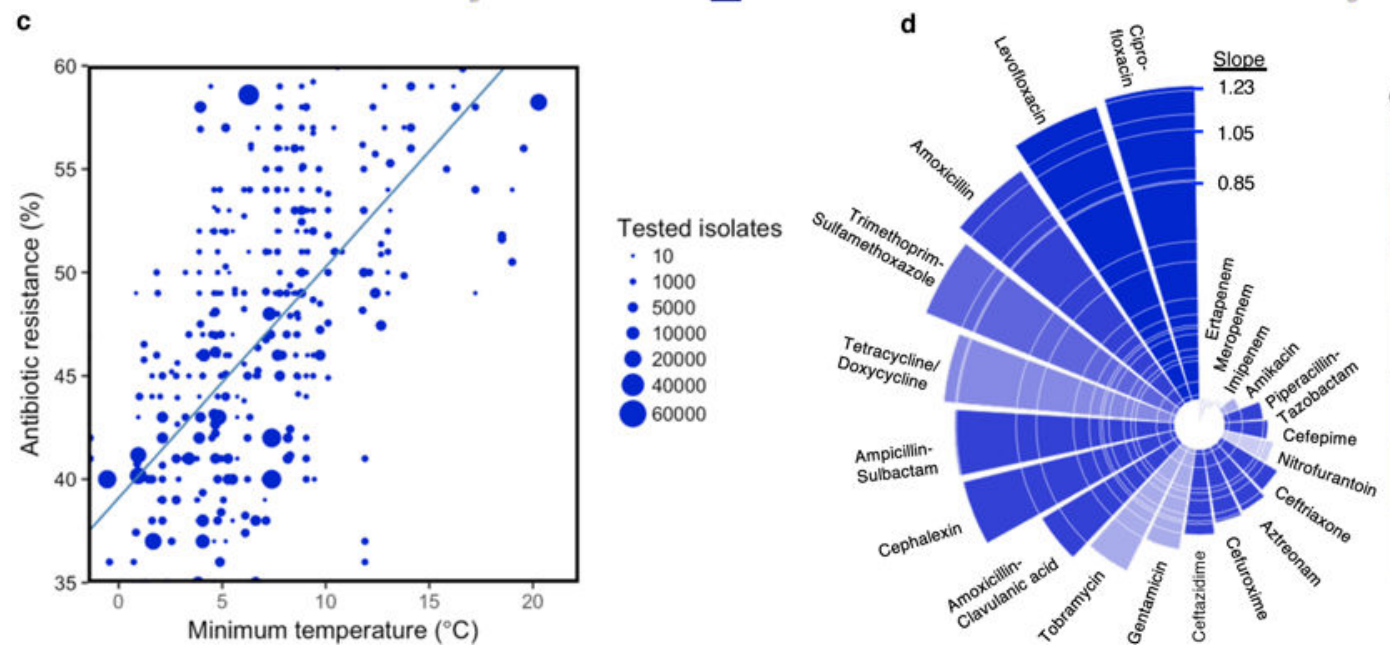

Class

Fluoroquinolones

Sulfa

Tetracyclines

Aminoglycosides

Hydantoins

Carbapenems

Beta-lactams/Monobactams

Figure 1. Antibiotic resistance increases with increasing temperature.

(A) A heatmap of mean normalized antibiotic resistance for $E$. coli for all antibiotics across the United States. (B) A heatmap of 30-year average minimum temperature $\left({ }^{\circ} \mathrm{C}\right)$ across the United States. (C) A scatter plot of antibiotic resistance versus minimum temperature $\left({ }^{\circ} \mathrm{C}\right)$ by acquisition type for $E$. coli and amoxicillin. Unadjusted weighted linear trend line is shown in blue. (D) Slope of unadjusted relationship (\% Resistance $\left./{ }^{\circ} \mathrm{C}\right)$ between minimum temperature and antibiotic resistance by antibiotic for $E$. coli. Antibiotic class coded by color shading. 


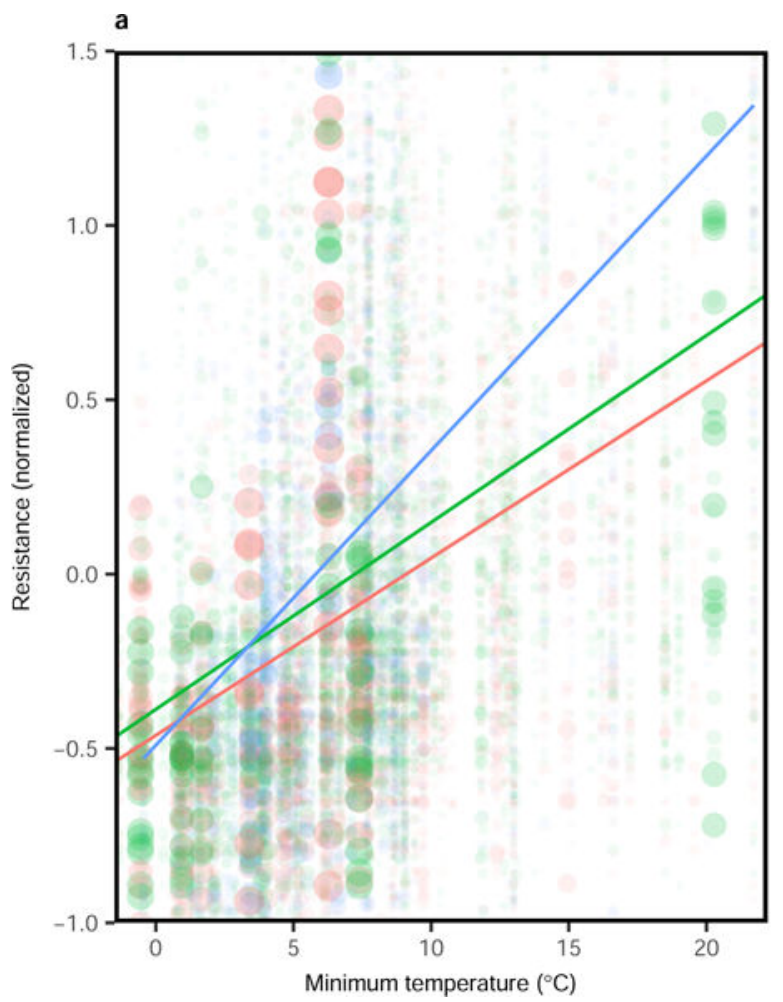

$$
\begin{aligned}
& \text { Year } \\
& -2013 \\
& -2014 \\
& -2015 \\
& \\
& \text { Tested isolates } \\
& 10 \\
& 1000 \\
& 5000 \\
& 10000 \\
& 20000 \\
& 40000 \\
& 60000
\end{aligned}
$$
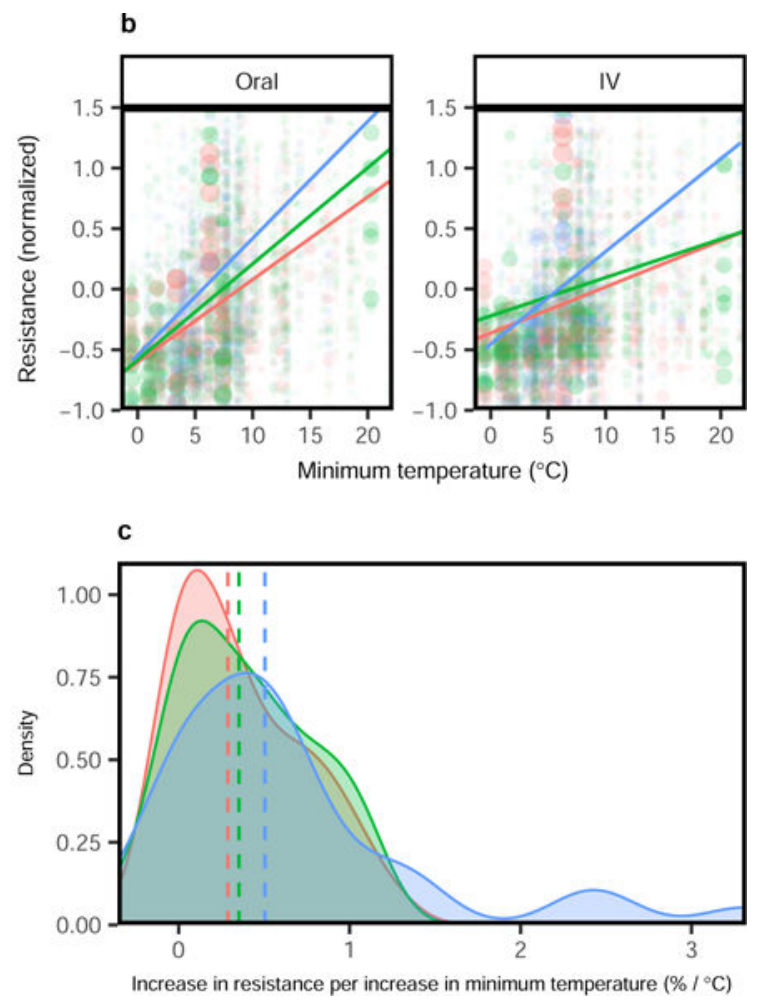

Figure 2. Change in the relationship between minimum temperature and antibiotic resistance over time.

(A) Mean normalized antibiotic resistance versus minimum temperature $\left({ }^{\circ} \mathrm{C}\right)$ for all pathogens and antibiotics, stratified by year (2013-2015). Unadjusted weighted linear relationships for years 2013-2015 are shown. (B) Mean normalized antibiotic resistance versus minimum temperature $\left({ }^{\circ} \mathrm{C}\right)$ for all pathogens and antibiotics, stratified by year and route (oral versus IV). (C) Density distributions of association measures (slopes) between antibiotic resistance and minimum temperature, stratified by time and with median densities (by year) marked by vertical dashed lines 


\section{Table 1}

Weighted multivariable regression analysis of evaluated predictors of antibiotic resistance.

\begin{tabular}{|c|c|c|c|c|}
\hline Bacteria & Variable & Unadjusted & Unadjusted* & Adjusted* \\
\hline \multirow[t]{10}{*}{ E. coli } & Minimum temp & 0.512 & 0.598 & 0.419 \\
\hline & & $(<0.0001)$ & $(<0.0001)$ & $(<0.0001)$ \\
\hline & Prescription rate & -- & 0.120 & 0.121 \\
\hline & & & $(<0.0001)$ & $(<0.0001)$ \\
\hline & Outpatient & -3.280 & -4.590 & -2.980 \\
\hline & & $(0.14)$ & $(0.09)$ & $(0.06)$ \\
\hline & Lab standard & -4.240 & -4.900 & -3.240 \\
\hline & & $(0.08)$ & $(0.06)$ & $(0.16)$ \\
\hline & Population density & 0.00027 & 0.00035 & 0.00027 \\
\hline & & 0.006 & $(<0.0001)$ & $(0.086)$ \\
\hline \multirow[t]{10}{*}{ K. pneumoniae } & Minimum temp & 0.341 & 0.397 & 0.219 \\
\hline & & $(<0.0001)$ & $(<0.0001)$ & $(<0.0001)$ \\
\hline & Prescription rate & -- & 0.030 & 0.034 \\
\hline & & & $(<0.0001)$ & $(<0.0001)$ \\
\hline & Outpatient & -2.640 & -3.006 & -2.880 \\
\hline & & $(0.17)$ & $(0.24)$ & $(0.17)$ \\
\hline & Lab standard & -1.760 & -2.220 & -0.371 \\
\hline & & $(0.17)$ & $(0.22)$ & $(0.75)$ \\
\hline & Population density & 0.00051 & 0.00063 & 0.0006 \\
\hline & & $(<0.0001)$ & $(<0.0001)$ & $(<0.0001)$ \\
\hline \multirow[t]{10}{*}{ S. aureus } & Minimum temp & 0.305 & 0.571 & 0.273 \\
\hline & & $(0.002)$ & $(0.004)$ & $(0.21)$ \\
\hline & Prescription rate & -- & 0.133 & 0.134 \\
\hline & & & $(<0.0001)$ & $(<0.0001)$ \\
\hline & Outpatient & -5.800 & -5.570 & -4.243 \\
\hline & & $(<0.0001)$ & $(<0.0001)$ & (0.0003) \\
\hline & Lab standard & -1.780 & -3.450 & -3.922 \\
\hline & & $(0.08)$ & $(0.08)$ & $(0.03)$ \\
\hline & Population density & 0.00014 & 0.00015 & 0.00005 \\
\hline & & $(0.15)$ & $(0.07)$ & $(0.69)$ \\
\hline \multirow[t]{9}{*}{ S. aureus ${ }^{* *}$} & Minimum temp & 0.405 & 0.762 & 0.514 \\
\hline & & $(<0.0001)$ & $(<0.0001)$ & $(<0.0001)$ \\
\hline & Prescription rate & -- & 0.068 & 0.066 \\
\hline & & & $(0.0006)$ & $(<0.0001)$ \\
\hline & Outpatient & -6.338 & -6.520 & -4.570 \\
\hline & & $(0.0005)$ & $(<0.0001)$ & $(0.01)$ \\
\hline & Lab standard & -2.305 & -4.910 & -3.840 \\
\hline & & $(<0.0001)$ & $(0.008)$ & $(0.01)$ \\
\hline & Population density & 0.0002 & 0.0002 & 0.00004 \\
\hline
\end{tabular}




\begin{tabular}{ccccc}
\hline Bacteria & Variable & Unadjusted & Unadjusted $^{*}$ & Adjusted $^{*}$ \\
\hline & $(0.035)$ & $(0.009)$ & $(0.27)$ \\
\hline
\end{tabular}

Unadjusted and adjusted multivariable regression parameters for historical (30-year) mean minimum temperature $\left({ }^{\circ} \mathrm{C}\right)$, antibiotic class specific prescription rate (annual prescriptions/1000 population), acquisition source (containing outpatient isolates), laboratory standard (CLSI vs. other/not reported), and population density (persons $/ \mathrm{mi}^{2}$ ). P-values are shown in parentheses ().

Restricted to subset of antibiotic susceptibilities for dates and classes of antibiotics corresponding to available prescribing rate data. **

Analysis with Penicillin susceptibility removed. 